\title{
WSO-UV field camera unit for comet and exoplanet observations
}

\author{
S. Sichevsky, E. Kanev, V.Shmagin and M.Sachkov \\ Institute of Astronomy of the Russian Academy of Sciences, 48 Pyatnitskaya \\ st. 119017, Moscow, Russia
}

Received: August 5, 2021; Accepted: November 25, 2021

\begin{abstract}
The World Space Observatory for Ultraviolet (WSO-UV) is a space observatory equipped with instrumentation for spectroscopy and for imaging in the UV spectral range. After the Hubble Space Telescope, WSO-UV will be the largest mirror telescope for UV astronomy. Thus, the WSO-UV space observatory will guarantee continuity of UV observation of comets and exoplanets.
\end{abstract}

Key words: space vehicles: instruments - ultraviolet: general - comets

\section{Introduction}

WSO-UV is a space observatory in geostationary orbit with an inclination of $\sim 40^{\circ}$. One has a $1.7-$ meter telescope capable of spectroscopy and direct imaging in the UV range of the spectrum (115 to $305 \mathrm{~nm}$ ). The nominal lifetime is 5 years with an expected extension of up to 10 years.

The main scientific purpose of WSO-UV is the spectroscopic observation of faint UV sources and high resolution UV imaging (Fossati et al., 2014; Sachkov, 2016; Sachkov et al., 2018b). WSO-UV includes the T-170M telescope designed to fit requirements of high angular resolution and maximum effective area in the $115 \mathrm{~nm}-305 \mathrm{~nm}$ range. It provides the solar avoidance angle about $40^{\circ}$, that is important for observations of comets at low angular distance from the Sun.

An additional tool has recently been proposed for WSO-UV - UVSPEX, a UV spectrograph, is designed to measure atomic hydrogen and oxygen in the exospheres of terrestrial exoplanets (Tavrov et al., 2018). Examination of the transit photometric curves of the exosphere can help differentiate the different types of rocky planets.

\section{Field Camera Unit}

One of main instruments of WSO-UV is Field Camera Unit (FCU) which has two channels: NUV (near ultraviolet channel) and FUV (vacuum or far ultraviolet channel):

- FUV: the working wavelength range no narrower than from 115 to $176 \mathrm{~nm}$; 
Table 1. NUV channel filters to study Earth-like exoplanets.

\begin{tabular}{llll}
\hline \hline Name & Type & Central wavelength, nm & FWHM, nm \\
\hline F255W & Wide-band filter & 255 & 50 \\
F336W & Wide-band filter & 336 & 50 \\
\hline \hline
\end{tabular}

- NUV: from 174 to $305 \mathrm{~nm}$.

The FUV channel has an MCP detector, which is supplied under the international agreement between Russia and Spain. The main characteristics of the FUV channel are as follows:

- Diffraction-quality imaging.

- Highly sensitive photon-counting mode.

- High temporal resolution.

The NUV channel has a CCD detector sensitive from 174 to $305 \mathrm{~nm}$ (with a possible expansion to $1000 \mathrm{~nm}$ ). The main characteristics of the NUV channel:

- Large field of view (FoV).

- Large dynamic range

Key scientific problems of FCU: study of planetary nebulae; UV behavior of a supernova; study of short-term (about $40 \mathrm{~ms}$ ) variable sources; astroseismology; exoplanetary atmospheres; protostellar jets; Galactic globular clusters and variables. For more details on the scientific problems of the FCU, see Sachkov et al. (2018a)

The study of comets is one of the most promising areas of astronomy for the next decade. UV-spectroscopy of comets in the 115-300 nm wavelength range plays an important role, since this part of the spectrum contains most of the resonance lines of atoms of molecules and ions. The large FoV and high sensitivity of the NUV channal of FCU will make WSO-UV the most efficient observatory to track comet evolution.

To study Earth-like exoplanets by transits photometry method, two ozone absorption bands filters will be used: 230-280 nm (Hartley band, F255W) and 310-360 nm (Huggins band, F336W).

\section{NUV channel: technical realisation of Hartley and Hug- gins bands}

NUV channel is located in the center of telescope's field of view. It provides high transmittance as it contains no additional optics except flat pick-off mirror. It 


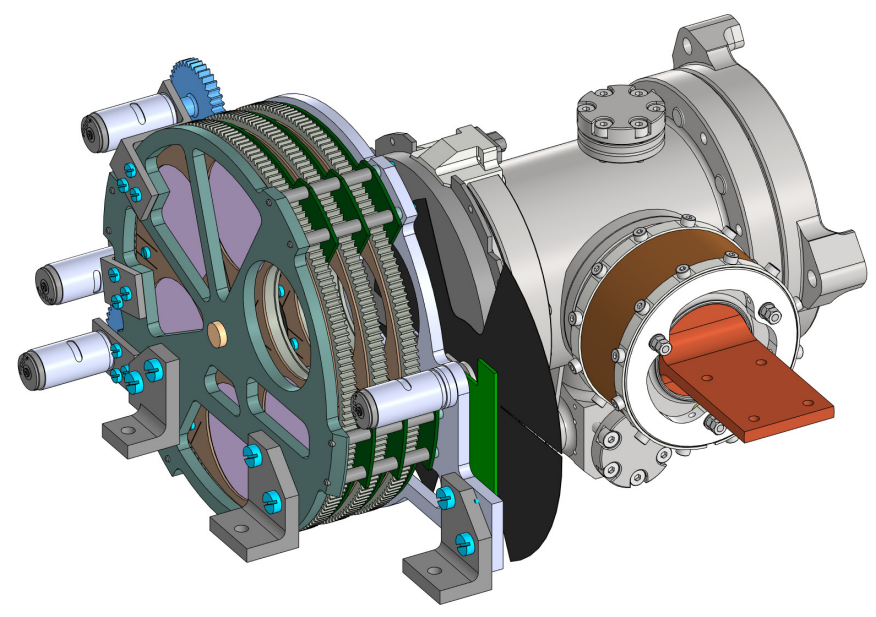

Figure 1. A general view of filter wheels of NUV channel.

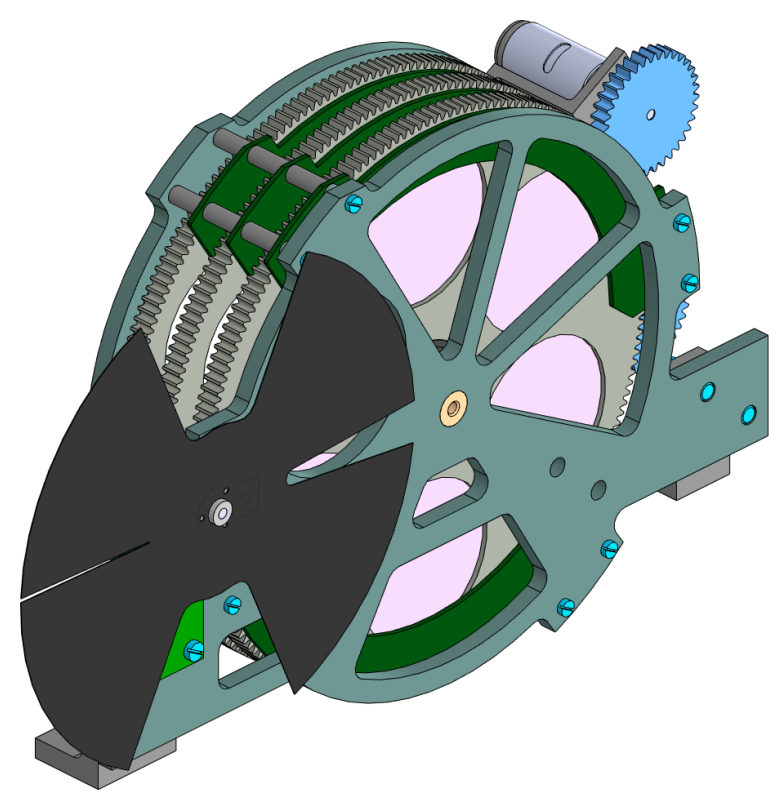

Figure 2. Full-turn shutter of NUV channel. 
is equipped with 3 filter wheels with 5 filters and 1 open slot in each one, so up to 15 filters can be used with possibility of combination (see Fig.1).

Full-turn shutter contains 2 open slots for long exposures of faint objects and 2 slits with different width for bright targets and flat-field calibration (see Fig.2).

\subsection{Conclusions}

The large FoV and high sensitivity of the NUV channel of FCU will make WSOUV the most efficient observatory to track comet evolution.

The WSO-UV space observatory, scheduled to launch in 2025, could become an important tool in cometary and explanetary UV research in the next decade.

Thus, The WSO-UV space observatorywill guarantee continuity of UV observation of comets and expoplanets.

Acknowledgements. Authors acknowledge the support of Ministry of Science and Higher Education of the Russian Federation under the grant 075-15-2020-780 (N13.1902.21.0039).

\section{References}

Fossati, L., Bisikalo, D., Lammer, H., Shustov, B., \& Sachkov, M., Major prospects of exoplanet astronomy with the World Space Observatory-UltraViolet mission. 2014, Astrophysics and Space Science, 354, 9, DOI: 10.1007/s10509-014-2027-3

Sachkov, M., Shustov, B., Gómez de Castro, A. I., et al., The new field camera unit imaging instrument onboard WSO-UV. 2018a, in Society of Photo-Optical Instrumentation Engineers (SPIE) Conference Series, Vol. 10699, Space Telescopes and Instrumentation 2018: Ultraviolet to Gamma Ray, ed. J.-W. A. den Herder, S. Nikzad, \& K. Nakazawa, 1069935

Sachkov, M. E., Spectral studies of comets in the ultraviolet range and prospects of the WSO-UV project in these studies. 2016, Solar System Research, 50, 294, DOI: $10.1134 / \mathrm{S} 0038094616040055$

Sachkov, M. E., Kartashova, A. P., \& Emel'yanenko, V. V., Prospects for spectral studies of comets: The World Space Observatory - Ultraviolet project. 2018b, Planetary Space Science, 164, 75, DOI: 10.1016/j.pss.2018.06.013

Tavrov, A., Kameda, S., Yudaev, A., et al., Stellar Imaging Coronagraph and Exoplanet Coronal Spectrometer: Two Additional Instruments for Exoplanet Exploration Onboard The WSO-UV 1.7 Meter Orbital Telescope. 2018, arXiv e-prints, arXiv:1810.07644 